\title{
Infant feeding practices within a large electronic medical record database
}

\author{
Emily Bartsch ${ }^{*}$, Alison L. Park², Jacqueline Young ${ }^{2}$, Joel G. Ray ${ }^{3}$ and Karen Tu ${ }^{4}$
}

\begin{abstract}
Background: The emerging adoption of the electronic medical record (EMR) in primary care enables clinicians and researchers to efficiently examine epidemiological trends in child health, including infant feeding practices.

Methods: We completed a population-based retrospective cohort study of 8815 singleton infants born at term in Ontario, Canada, April 2002 to March 2013. Newborn records were linked to the Electronic Medical Record Administrative data Linked Database (EMRALD ${ }^{T M}$ ), which uses patient-level information from participating family practice EMRs across Ontario. We assessed exclusive breastfeeding patterns using an automated electronic search algorithm, with manual review of EMRs when the latter was not possible. We examined the rate of breastfeeding at visits corresponding to 2, 4 and 6 months of age, as well as sociodemographic factors associated with exclusive breastfeeding.

Results: Of the 8815 newborns, 1044 (11.8\%) lacked breastfeeding information in their EMR. Rates of exclusive breastfeeding were $39.5 \%$ at 2 months, 32.4\% at 4 months and $25.1 \%$ at 6 months. At age 6 months, exclusive breastfeeding rates were highest among mothers aged $\geq 40$ vs. $<20$ years (rate ratio [RR] 2.45, 95\% confidence interval [Cl] 1.62-3.68), urban vs. rural residence (RR 1.35, 95\% Cl 1.22-1.50), and highest vs. lowest income quintile (RR $1.18,95 \% \mathrm{Cl} 1.02-1.36$ ). Overall, immigrants had similar rates of exclusive breastfeeding as non-immigrants; yet, by age 6 months, among those residing in the lowest income quintile, immigrants were more likely to exclusively breastfeed than their non-immigrant counterparts (RR 1.43, 95\% Cl 1.12-1.83).
\end{abstract}

Conclusions: We efficiently determined rates and factors associated with exclusive breastfeeding using data from a large EMR database.

Keywords: Breastfeeding, Formula feeding, Infant nutrition, Electronic medical record, EMR

\section{Background}

Breastmilk is recognized as the optimal form of infant nutrition, and has been shown to confer significant benefit to both the mother and infant [1-3]. For instance, exclusive breastfeeding is protective against infection and gastroenteritis in infancy [2, 3], and may prevent the development of asthma [2] and childhood obesity [4] in the long-term. Despite the benefits, and consequent recommendations from the World Health Organization (WHO) [5] and the Canadian Paediatric Society [6], according to the 2009 Maternity Experiences Survey (MES), only $14 \%$ of Canadian infants are exclusively breastfed at 6 months [7]. The 2009 Canadian Community Health Survey (CCHS) reported rates of

\footnotetext{
* Correspondence: emily.bartsch@mail.utoronto.ca

'University of Toronto, Toronto, Canada

Full list of author information is available at the end of the article
}

exclusive breastfeeding in the first 6 months ranging from $22 \%$ to $33 \%$ from 2011 to 2014 [8]. However, the MES used telephone interviews to gather information from a stratified random sample, while the CCHS employs a cross-sectional, voluntary questionnaire.

Preterm infants are less likely to be breastfed $[9,10]$. Of note, infants of immigrant women to Canada - who comprise $35 \%$ of all births in Ontario [11] - may not have significantly different rates of breastfeeding than those of Canadian-born women [12]. Worldwide, the WHO reports that return to work is the largest barrier to breastfeeding [13].

The increasing use of electronic medical records (EMR) in primary care provides an opportunity to efficiently explore feeding practices in large general populations, perhaps with less bias than may occur in self-report surveys or registries. 
We set out to determine rates of exclusive breastfeeding and sociodemographic factors that relate to exclusive breastfeeding of Ontario infants.

\section{Methods}

We performed a retrospective population-based cohort study using administrative datasets linked using unique encoded identifiers and analyzed at the Institute for Clinical Evaluative Sciences (ICES). We considered singleton term infants born in an Ontario hospital between April 1, 2002 and March 31, 2013, whose mother was an Ontario resident at the time of the birth. Maternal-newborn pairs were identified from the ICES MOMBABY dataset, which links the inpatient records of delivering mothers and their newborns in the Canadian Institute for Health Information (CIHI) Discharge Abstract Database (DAD), housed at ICES. Our study cohort was further limited to infants who had at least 1 postnatal visit with a family physician within 190 days of age in the Electronic Medical Record Administrative data Linked Database $\left(\right.$ EMRALD $\left.^{\mathrm{mm}}\right)$, also housed at ICES. The EMRALD ${ }^{\mathrm{mi}}$ dataset was created using data from participating family practice EMRs across Ontario [14]. Specific to our study, EMRALD ${ }^{\text {tw }}$ contains data for wellbaby visits to family physicians, including the date of each visit, the infant's corresponding age, anthropometric measures, feeding practices, developmental milestones and physical exams. Multi-fetal pregnancies were excluded as they are more prone to indicate preterm birth, small-for-gestational age, and specialized pediatric postnatal care.

To obtain information about feeding practices, we formulated an algorithm combining free text searches and structured field searches from the Rourke Baby Record, which is described at http://www.rourkebabyrecord.ca/pdf/RBR\%202017\%20Ontario\%20English\%20\%20Black\%20171004.pdf and http://rourkebabyrecord.ca/default.asp. The Rourke Baby Record is a standardized and commonly used method for family physicians in Canada to record well baby visits in newborn and infant medical records. The content of the search algorithm is listed in the Additional file 1. The electronic search algorithm was used to abstract information about mode of infant feeding from all EMRs with a Rourke Baby Record. To find Rourke Baby Records the search algorithm looked in the free text progress notes made by the family physician for mention of a Rourke form. The name of the form can vary, so as many variations were accounted for, such as "Rourke", "Well Baby Visit", "Well Baby Check Up", "Newborn Visit", "1 month visit", etc. If a Rourke Baby Record was found, the algorithm to search for documentation of feeding was then applied. Those EMRs without a Rourke Baby Record were manually abstracted for similar details about infant feeding. We included records up to 750 days of age in order to capture historical information on type and duration of feeding recorded in non-Rourke entries, as well as Rourke Baby Records with exclusive breastfeeding documented beyond 6 months, from which we inferred exclusive breastfeeding at earlier time points.

Three trained abstractors performed the manual EMR abstraction. The initial charts were also reviewed by a content expert (ALP) to correct for any inconsistencies. Both intra and inter-rater reliability were assessed for 5 $\%$ of the charts.

Typically, well baby visits to primary care providers occur shortly after birth and at 2 months, 4 months and 6 months, according to the Ontario infant immunization schedule [15]. As such, we chose these time points to assess the rates of exclusive breastfeeding. The denominators for the rates at 2, 4 and 6 months included infants with any visit at $\geq 60$ days, $\geq 122$ days, and $\geq 182$ days of age, respectively. We chose the visit closest to but not preceding the target age for each time point. For children who did not have visits at all three time points, we estimated feeding status based on that documented at future visits. For example, if a child had a visit at 60 days then their 2 month feeding status was determined from that visit. If, however, their next visit was not until 182 days or more, then we determined if they were currently exclusively breastfeeding (from a Rourke record) or were previously exclusively breastfeeding (from a progress note) and we inferred that they were exclusively breastfeeding at 4 and 6 months.

To assess possible misclassification of exclusive breastfeeding as a result of inferring feeding status from future visits, we performed a complete case analysis of infants who had documented feeding at all three time points. Exclusive breastfeeding duration was calculated based on the date of birth and the date of the latest visit with confirmed exclusive breastfeeding.

Newborns to immigrant mothers were determined by linkage to the federal Immigration, Refugees and Citizenship Canada Permanent Resident Database held at ICES, which has records for permanent residents who immigrated to Ontario from 1985 to 2012. Neighborhood income quintile $(\mathrm{Q})$ was determined by residential postal code at the time of birth, derived from Statistics Canada census data. Rurality was determined by the Registered Persons Database (RPDB) and maternal age at birth, parity and birthweight were determined by linkage with the MOMBABY database.

Rate ratios (RR) and 95\% confidence intervals (CI) were calculated for each characteristic, comparing each group to that with the lowest rate (the reference group).

Statistical analysis was performed using SAS for UNIX, Version 9.4 (SAS Institute, Cary, NC), and EMR data 
analysis was performed in SQL Server 2012. The study was approved by the Sunnybrook Health Sciences Centre Research Ethics Board.

\section{Results}

We identified 8815 singleton term newborns with a birth record in MOMBABY and at least 1 postnatal visit in EMRALD $^{\text {tw }}$ before 190 days. The total number of visits for these infants was 110,794. In our study, 25,230 visits were included from infants with a Rourke Baby Record, and 54,856 visits were abstracted from the chart. The infants and mothers in EMRALD ${ }^{\mathrm{m} w}$ were similar to those across Ontario in terms of characteristics such as age and parity, however mothers in EMRALD ${ }^{\mathrm{Tw}}$ were somewhat more likely to be a rural resident and Canadianborn, and less likely to be South or East Asian born and in lower income quintiles (Table 1). Of the 8815 infants in EMRALD $^{\mathrm{ma}}, 7051$ (80.0\%) had any Rourke Baby Record, while 1764 (20.0\%) did not (Fig. 1). Among infants with a Rourke Baby Record, we identified infant feeding using the automated search algorithm for 4955 (70.3\%) of patients. For the remainder of infants, manual abstraction was performed, wherein we identified feeding for 2816 additional infants. Feeding remained undetermined for 1044 (11.8\%) of infants.

The Cohen's kappa statistic for inter-rater reliability of infant feeding classification was high (0.936). Based on all the automated and manually abstracted feeding results, $45.9 \%$ of infants were classified as exclusively breastfed, $14.5 \%$ exclusively formula-fed, and $27.7 \%$ mixed breastfed and formula-fed. The rates of breastfeeding were higher among infants in the automatically abstracted group than those of the manually abstracted group at 2, 4, and 6 months.

Rates of exclusive breastfeeding were $39.5 \%$ at 2 months, $32.4 \%$ at 4 months, and $25.1 \%$ at 6 months (Table 2). In a complete case analysis of 3959 infants who had visits at all three time points, the rates of exclusive breastfeeding did not significantly differ (data not shown). Breastfeeding rates were higher with greater maternal age, such that women over 30 were more than twice as likely as women under 20 to exclusively breastfeed at any time. Exclusive breastfeeding was more prevalent among urban than rural residents, women living in higher income neighbourhoods, and those who delivered in 2008 onward. Exclusive breastfeeding was

Table 1 Characteristics of all liveborn singleton infants and their mothers, born in an Ontario hospital between April 1, 2002 and March 31, 2013. Shown are births in the MOMBABY dataset vs. those concomitantly available in the EMRALD ${ }^{\text {TM }}$ database

\begin{tabular}{|c|c|c|c|}
\hline Characteristic & $\begin{array}{l}\text { MOMBABY dataset } \\
(N=1,204,042)\end{array}$ & $\begin{array}{l}\text { EMRALD }^{\text {TM }} \text { dataset } \\
(N=8,815)\end{array}$ & Standardized difference \\
\hline Mean (SD) age, years & $29.9(5.5)$ & $30.2(5.3)$ & -0.1 \\
\hline Median (IQR) parity & $1(1)$ & $1(1)$ & 0.0 \\
\hline Rural residence, $n$ (\%) & $125,850(10.5)$ & $2151(24.4)$ & -0.4 \\
\hline \multicolumn{4}{|l|}{ Mother's World region of birth, $n(\%)$} \\
\hline Canada & $901,188(74.9)$ & 7719 (87.6) & -0.3 \\
\hline Europe/Western & $47,586(4.0)$ & $358(4.1)$ & 0.0 \\
\hline African/Caribbean & $38,508(3.2)$ & $132(1.5)$ & 0.1 \\
\hline Middle East/N Africa & $30,679(2.6)$ & $120(1.4)$ & 0.1 \\
\hline Latin America & $23,076(1.9)$ & $121(1.4)$ & 0.0 \\
\hline South Asia & $94,458(7.9)$ & $133(1.5)$ & 0.3 \\
\hline East Asia & $68,467(5.7)$ & $227(2.6)$ & 0.2 \\
\hline Unknown & $80(<0.1)$ & $\leq 5(<0.1)$ & 0.0 \\
\hline \multicolumn{4}{|l|}{ Income quintile (Q), n (\%) } \\
\hline Q1 & $265,136(22.0)$ & $1540(17.5)$ & 0.1 \\
\hline Q2 & $240,322(20.0)$ & $1660(18.8)$ & 0.0 \\
\hline Q3 & $244,706(20.3)$ & $1922(21.8)$ & 0.0 \\
\hline Q4 & $249,328(20.7)$ & $1900(21.6)$ & 0.0 \\
\hline Q5 & $199,110(16.5)$ & $1756(19.9)$ & -0.1 \\
\hline Unknown & $5440(0.5)$ & $37(0.4)$ & 0.0 \\
\hline Mean (SD) birthweight, g & $3461(472)$ & $3490(464)$ & -0.1 \\
\hline Mean (SD) gestational age, weeks & $39.2(1.1)$ & $39.3(1.1)$ & -0.1 \\
\hline Infant male sex, $n(\%)$ & $610,661(50.7)$ & $4527(51.4)$ & 0.0 \\
\hline
\end{tabular}




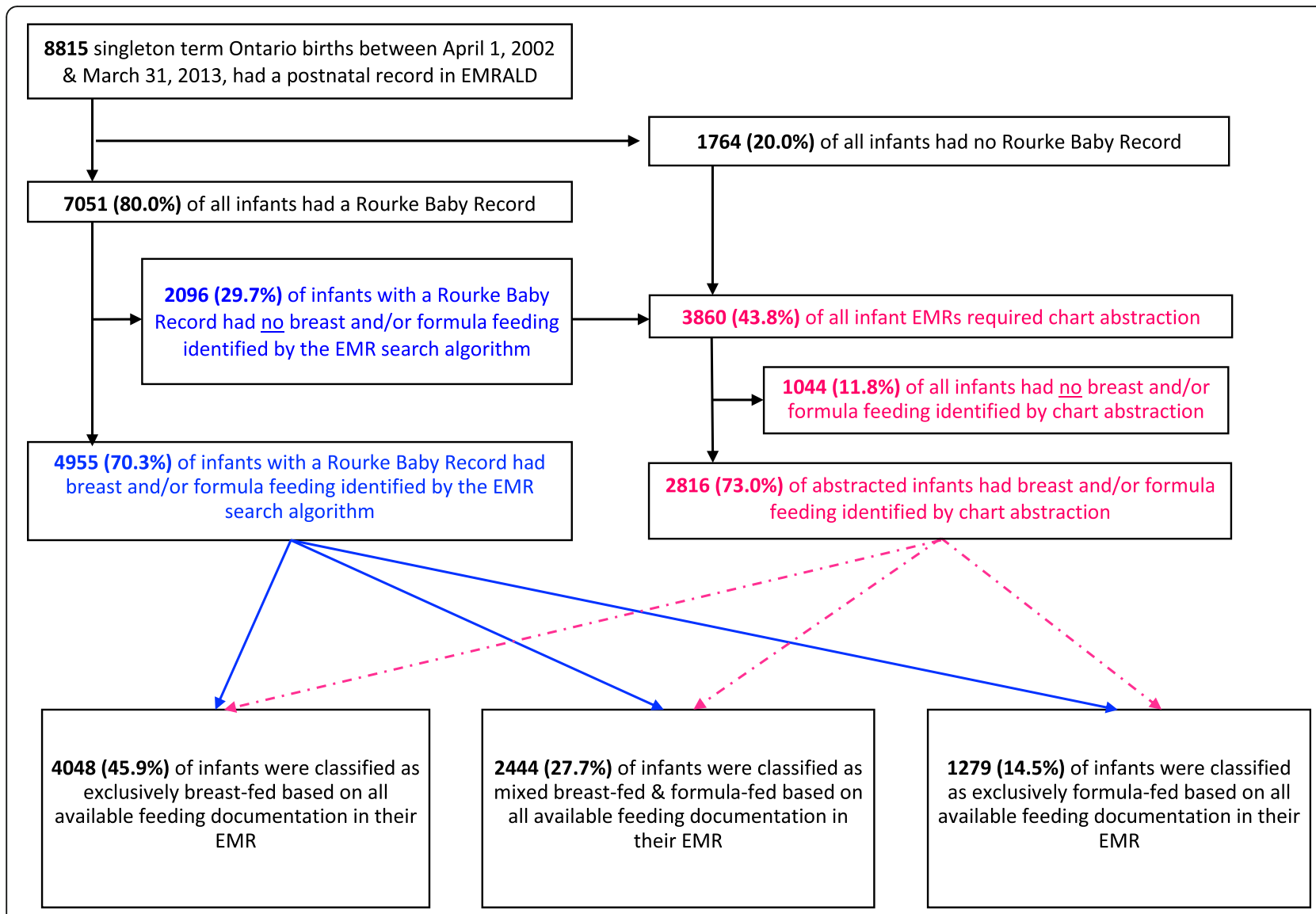

Fig. 1 Derivation of the infant feeding cohort and validation cohort for the electronic medical record (EMR) search algorithm

not associated with parity, birthweight, or immigrant status; however, immigrant women living in the lowest income areas had significantly higher rates of breastfeeding than non-immigrant women living in the lowest income neighbourhood (Table 2).

\section{Discussion}

We examined exclusive breastfeeding rates among term singleton infants within a primary care EMR database. Previously, results from the MES, which used telephone administered questions, reported that $90 \%$ of women in Ontario intended to initiate breastfeeding, and 90\% did so [16]. At 6 months, approximately half of women reported continuation of some form of breastfeeding, while under $15 \%$ were exclusively breastfeeding [7]. Using a questionnaire survey method, the CCHS found that approximately $27 \%$ of Ontario women reported exclusively breastfeeding at 6 months [8]. Like the CCHS, our current study found that the rate of exclusive breastfeeding at 6 months was $25 \%$. Our results may be more representative of the general population, as they were taken from family medicine records of patients distributed throughout Ontario. Furthermore, by evaluating rates of breastfeeding over several time points, we observed a decrease in rates over time, especially between 4 and 6 months of age. At 2 months, $40 \%$ of infants were exclusively breastfed - a marked decrease from the $90 \%$ who initiate breastfeeding at birth [16].

As the uptake of EMRs continues to increase among primary care providers, data becomes more easily accessible, and the process of characterizing large cohorts becomes more efficient. Our study represents a large cohort of nearly 9000 infants - the largest study to date on breastfeeding in Ontario. The search algorithm we developed combined lists of free text terms and analysis of structured Rourke Baby Record fields. Although the search algorithm located feeding information for over half of the infants, over $40 \%$ further required manual chart abstraction, which is costly and time consuming. However the data developed here may be utilized for future studies that incorporate more sophisticated text mining and machine learning methods in order to allow for automated determination of breastfeeding rates in an automated time- and cost-efficient fashion. This will be beneficial for assessing changes in feeding practices over time, and the impact of breastfeeding promotion initiatives.

A few limitations herein need be acknowledged. Even after completing the manual chart abstraction, no feeding 


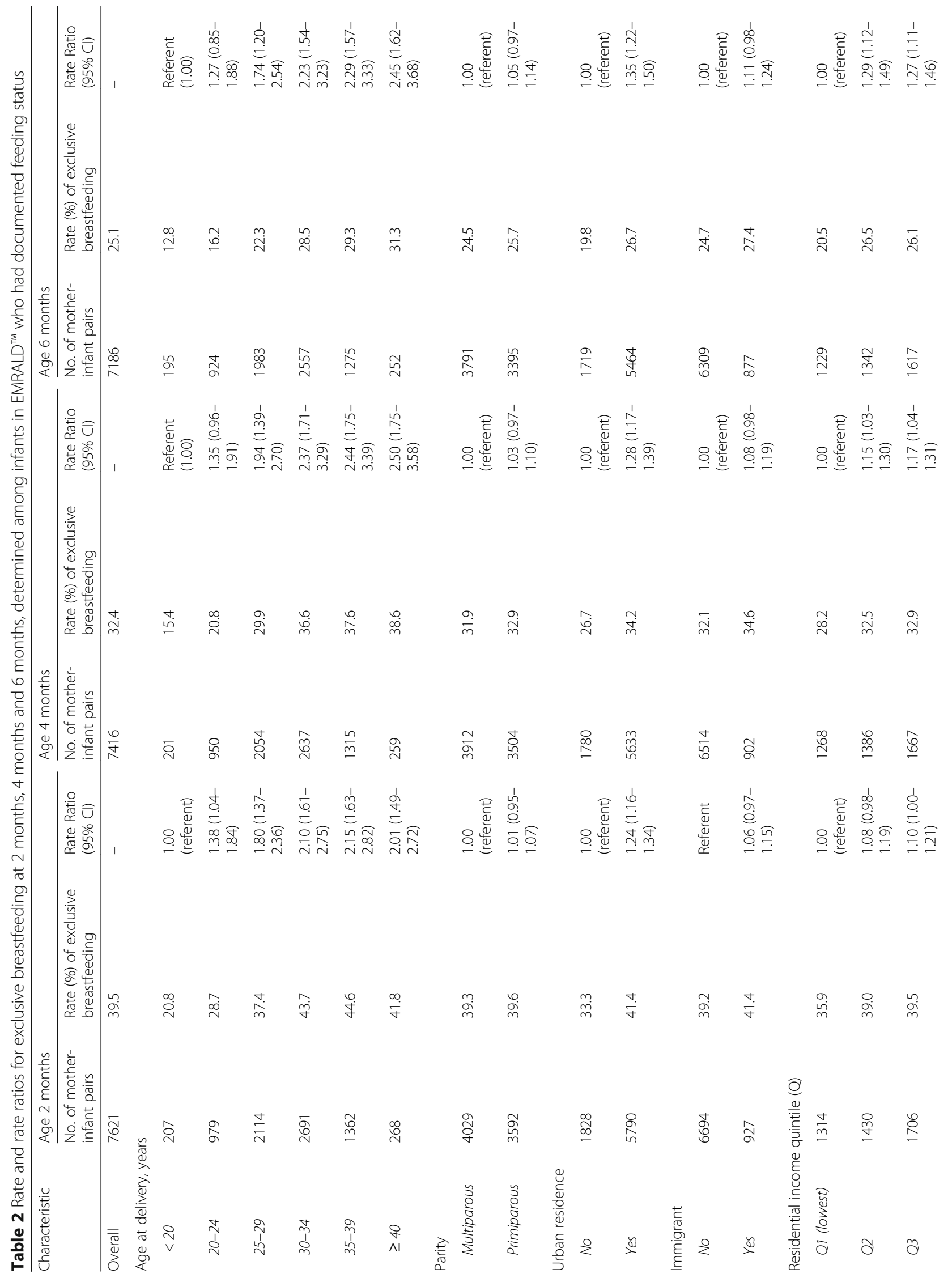




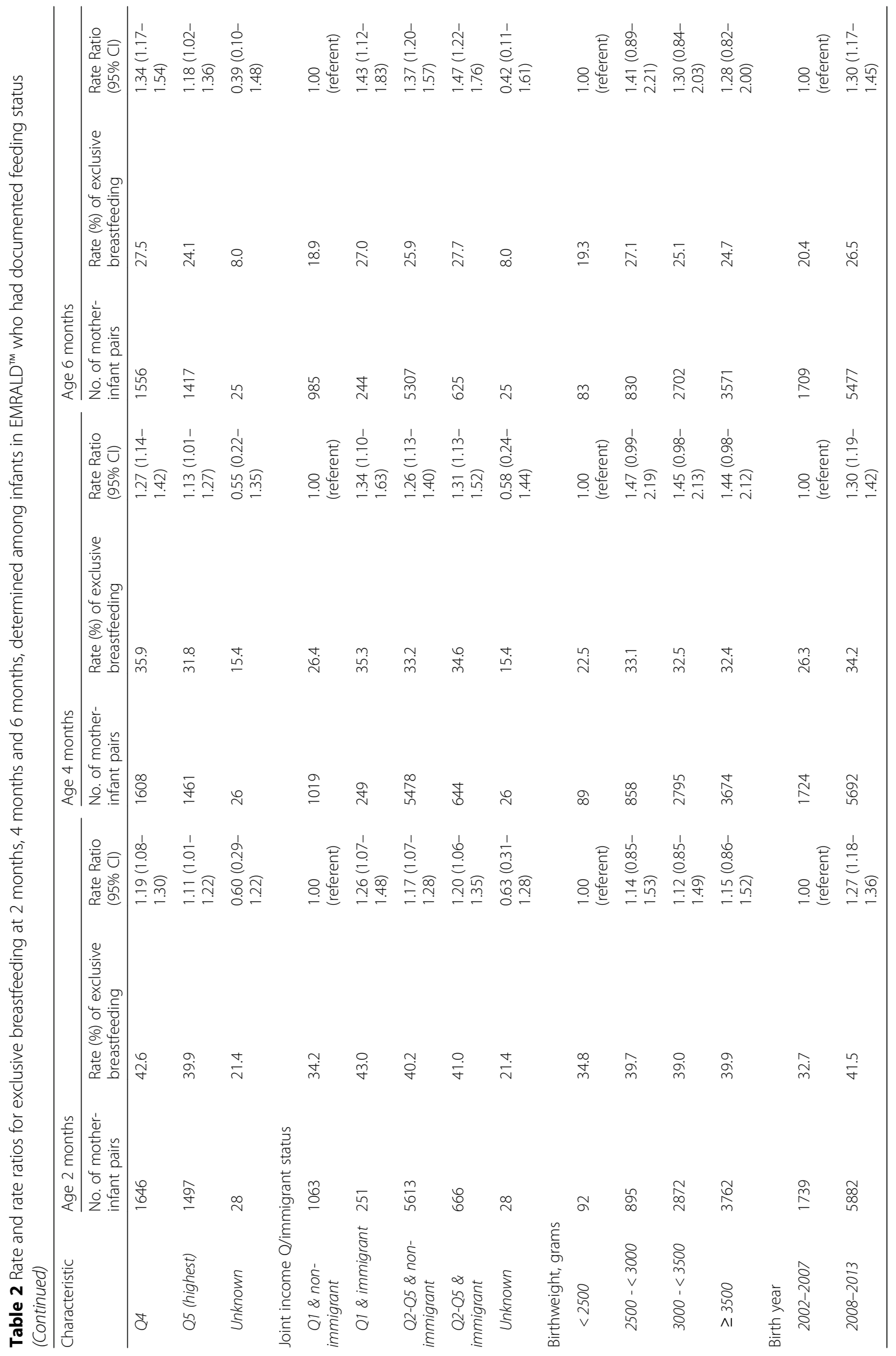


information could be obtained for over 1000 records. This suggests that there are inconsistencies in the way that EMRs, and standardized forms like the Rourke Baby Record, are completed. This highlights some challenges in using EMR data for secondary purposes. A second limitation of using EMR data was the resultant discrepancy in rates of breastfeeding between the infants in the automatic abstraction group compared to the manual abstraction group; however, the higher rate of breastfeeding with the automated algorithm was expected, given that all charts were put through an automated data extraction as an initial pass. Third, although the patients in EMRALD ${ }^{\mathrm{mm}}$ appeared to be ethnically diverse, mothers from South Asia and East Asia were under-represented, and rural dwelling women were over-represented in EMRALD ${ }^{\mathrm{m}}$, compared to the entire population of Ontarians. Despite its differences from the Ontario population, EMRALD ${ }^{\text {max }}$ may be the most closely representative dataset available, as the data do not come from a specialty practice, and comprises multiple clinics and physicians across Ontario. Last, we were unable to assess the impact of maternal education or employment on exclusive breastfeeding rates, as this information was not available in our data.

\section{Conclusions}

Among infants registered within a large primary care EMR, the rate of exclusive breastfeeding declined from $40 \%$ to $25 \%$ between two and 6 months of age. Exclusive breastfeeding was more likely among mothers who were older and residing in an urban and higher income quintile neighbourhood, and among immigrant women living in a lower income quintile neighborhoods. Our study suggests that primary care EMR data can be used to assess breastfeeding practices in large populations.

\section{Additional file}

Additional file 1: Terminology used to identify feeding status in an automated fashion. Broadly, the search query contained distinct terms related to breastfeeding, formula feeding, and the Rourke Baby Record. Each of these categories was then subdivided with the terms below comprising the final algorithm to classify newborn feeding status. (DOCX $101 \mathrm{~kb}$ )

\section{Abbreviations}

CCHS: Canadian community health survey; $\mathrm{ClHI}$ : Canadian institute for health information; DAD: Discharge abstract database; EMR: Electronic medical record; EMRALD ${ }^{\mathrm{T}}$ : Electronic medical record administrative data linked database; ICES: Institute for clinical evaluative sciences; MES: Maternity experiences survey; MOHLTC: Ministry of health and long-term care; RPDB: Registered persons database; WHO: World health organization

\section{Acknowledgements}

Chart abstraction was performed by Inderjit Kaur, Kangrui Lin, and Soudeh Taghdiri.

\section{Funding}

This work was supported by a grant from the Canadian Institutes of Health Research (CIHR). JGR holds a Canadian Institutes for Health Research Chair in Reproductive and Child Health Services and Policy Research. The funder had no role in study design, data collection and analysis, decision to publish, or preparation of the manuscript. KT is supported by a research scholar award from the Department of Family and Community Medicine at the University of Toronto.

This study was supported by the Institute for Clinical Evaluative Sciences (ICES), which is funded by an annual grant from the Ontario Ministry of Health and Long-Term Care (MOHLTC). The opinions, results and conclusions reported in this paper are those of the authors and are independent from the funding sources. No endorsement by ICES or the Ontario MOHLTC is intended or should be inferred. Parts of this material are based on data and information compiled and provided by $\mathrm{ClHI}$. However, the analyses, conclusions, opinions and statements expressed herein are those of the author, and not necessarily those of $\mathrm{ClHI}$.

\section{Availability of data and materials}

The data set from this study is held securely in coded form at the Institute for Clinical Evaluative Sciences (ICES). While data sharing agreements prohibit ICES from making the data set publicly available, access may be granted to those who meet pre-specified criteria for confidential access. The full dataset creation plan is available from the authors upon request.

\section{Authors' contributions}

$E B, A L P, K T, J Y$, and JGR conceived the study. ALP and JY performed the analyses. All authors contributed to the interpretation of the results, writing of the manuscript and approval of the final version.

Ethics approval and consent to participate

This study was approved by the institutional review board at Sunnybrook Health Sciences Centre, Toronto, Canada. Anonymity of participants included in the study was ensured through the use of administrative datasets analyzed at the Institute for Clinical Evaluative Sciences (ICES), which were linked using unique encoded identifiers. Consent to participate was not necessary because the data used in this study was accessed via a database. The authors of this study were authorized by ICES to access and analyse the datasets in order to complete the study.

\section{Consent for publication}

Not applicable.

\section{Competing interests}

The authors declare that they have no competing interests.

\section{Publisher's Note}

Springer Nature remains neutral with regard to jurisdictional claims in published maps and institutional affiliations.

\section{Author details}

${ }^{1}$ University of Toronto, Toronto, Canada. ${ }^{2}$ Institute for Clinical Evaluative Sciences, Toronto, Canada. ${ }^{3}$ Departments of Medicine, Health Policy Management and Evaluation, and Obstetrics and Gynecology St. Michael's Hospital, University of Toronto, Toronto, Canada. ${ }^{4}$ Department of Family and Community Medicine, and Health Policy Management and Evaluation, Toronto Western Hospital Family Health Team, University of Toronto, Toronto, Canada.

Received: 30 January 2017 Accepted: 15 December 2017

Published online: 02 January 2018

References

1. Kramer MS, Kakuma R. Optimal duration of exclusive breastfeeding. Cochrane Database Syst Rev. 2012;(8):Cd003517.

2. Ip S, Chung M, Raman G, Chew P, Magula N, DeVine D, et al. Breastfeeding and maternal and infant health outcomes in developed countries. Evidence report/technology assessment. 2007 Apr;153:1-186.

3. Dewey K. Guiding principles for complementary feeding of the breastfed child. 2004 [cited 2016 October 10]; Available from: http://www.who.int/ nutrition/publications/infantfeeding/a85622/en/index.html 
4. National Guideline, Clearinghouse. Primary prevention of childhood obesity, second edition. 2014.

5. World Health Organization. The optimal duration of exclusive breastfeeding report of the expert consultation. World Health Organization; 2001.

6. Boland M. Exclusive breastfeeding should continue to six months. Paediatr Child Health. 2005;10(3):148.

7. Public Health Agency of Canada. What mothers say: the Canadian maternity experiences survey. Ottawa: PHAC; 2009

8. Statistics Canada. Breastfeeding practices by province and territory (percent). 2011 [cited 2016 October 13]; Available from: http://www.statcan. gc.ca/tables-tableaux/sum-som/101/cst01/health92b-eng.htm

9. McDonald SD, Pullenayegum E, Chapman B, Vera C, Giglia L, Fusch C, et al. Prevalence and predictors of exclusive breastfeeding at hospital discharge. Obstet Gynecol. 2012;119(6):1171-9.

10. Lutsiv O, Giglia L, Pullenayegum E, Foster G, Vera C, Chapman B, et al. A population-based cohort study of breastfeeding according to gestational age at term delivery. J Pediatr. 2013;163(5):1283-8.

11. Immigration and Ethnoculutural Diversity in Canada. 2013 [cited August 3, 2016]; Available from: https://www12.statcan.gc.ca/nhs-enm/2011/as-sa/99010-x/99-010-x2011001-eng.cfm

12. Wilson-Mitchell K, Rummens JA. Perinatal outcomes of uninsured immigrant, refugee and migrant mothers and newborns living in Toronto, Canada. Int J Environ Res Public Health. 2013;10(6):2198-213.

13. Heymann J, Raub A, Earle A. Breastfeeding policy: a globally comparative analysis. Bull World Health Organ. 2013;91(6):398-406.

14. Tu K, Widdifield J, Young J, Oud W, Ivers NM, Butt DA, et al. Are family physicians comprehensively using electronic medical records such that the data can be used for secondary purposes? A Canadian perspective. BMC medical informatics and decision making. 2015;15:67.

15. Ontario Ministry of Health and Long-Term Care. Ontario's Publicly Funded Immunization Schedules. [cited 2016 October 18]; Available from: http:/ www.health.gov.on.ca/en/pro/programs/immunization/schedule.aspx

16. Chalmers B, Levitt C, Heaman M, O'Brien B, Sauve R, Kaczorowski J. Breastfeeding rates and hospital breastfeeding practices in Canada: a national survey of women. Birth. 2009:36(2):122-32.

\section{Submit your next manuscript to BioMed Central and we will help you at every step:}

- We accept pre-submission inquiries

- Our selector tool helps you to find the most relevant journal

- We provide round the clock customer support

- Convenient online submission

- Thorough peer review

- Inclusion in PubMed and all major indexing services

- Maximum visibility for your research

Submit your manuscript at www.biomedcentral.com/submit

Biomed Central 